\title{
A General Method of Constructing Layout with Single Factorial Effect Confounded in $\boldsymbol{p}^{n}$ Factorial Experiments
}

\author{
Md. Kamrul Hasan Khan and M A Jalil* \\ Department of Statistics, Biostatistics and Informatics, Dhaka University, Dhaka - 1000, Bangladesh
}

(Received: 10 June 2013; Accepted: 3 November 2013)

\begin{abstract}
A general method of constructing layout with single factorial effect confounded in $p^{n}$ factorial experiments is proposed. It becomes easier to construct the design of confounding a single factor in a $p^{n}$ factorial experiment, especially when the number of factors as well as the number of levels becomes larger.
\end{abstract}

Keywords: Adjustment Factor, Adjustment Vector, Confounding.

\section{Introduction}

When the number of factors as well as the number of levels of each factor is large, it becomes difficult to make homogeneous plots in practice. In such situations, we are bound to use a limited number of homogeneous plots to analyze the factorial effects. As a result, some factorial effects or interactions will be mixed up with block effect, i.e. confounding.

Bose and Kishan ${ }^{1}$, Bose ${ }^{2}$ described the construction of $p^{n}$ factorial design using finite geometries. The treatments are represented by $n$-tuples $\left(a_{1}, a_{2}, \ldots, a_{n}\right)$ where $a_{i}$ are elements of $G F(p)$. The method is available only when $p$ is prime or prime power. Fisher ${ }^{3}$ discussed a method to develop the connection of the subject with that of Abelian groups, to prove a general proposition connecting the minimal size of block required with the number of factors involved, and to supply a catalogue of systems of confounding available up to fifteen factors. A system of simultaneous confounding in $2^{n}$ factorial experiment has been described, where an intrablock subgroup is constructed with the common elements taken from the factorial effects of two incomplete blocks, each confounded with a single factorial effect ${ }^{4,5}$. Das ${ }^{6}$ described an equivalent method of Bose $^{2}$ in which some of the treatment factors are designated as basic factors and the others as added factors. Levels of added factors are derived by combination of levels of the basic factors over $G F(p)$. S. C. Cotter ${ }^{7}$ proposed a general method of confounding for symmetrical factorial experiments. This paper considered the problem of conducting a $p^{n}$ factorial experiment in blocks size $p^{1}$. These designs can be constructed for all values of $p$, although for certain values better confounding patterns are available. Also analysis of these designs was given, showing which components of the sum of squares were confounded with blocks. John and Dean ${ }^{8}$ described the construction of a particular class of single replicate block designs, which they call generalized cyclic designs. The essential feature of the method is that the $n$-tuples giving the treatments of a particular block constitute an Abelian group, the intrablock subgroup. Patterson ${ }^{9}$ described a general computer algorithm, called DSIGN, in which levels of treatment factors are derived by linear combinations of levels of plot and block factors. The method provides finite-field, generalized cyclic and other designs. Mallick, S. A. ${ }^{10,11}$ developed two systems of designing factorial effects with simultaneous confounding of two effects, one for $3^{n}$ and other for $4^{n}$ - factorial experiments. In these systems of simultaneous confounding, the combination of levels was based on some manipulating manner. Jalil, et. al. ${ }^{12}$ developed a matrix method of designing a single factorial effect confounded in a $p^{n}$ - factorial experiment, where the level combinations are obtained by matrix operations of the levels. This method is applicable only when $p$ is prime. In this article, we make moderation in taking adjustment factor using which we can construct a plan for a factorial effect to be confounded in a $p^{n}$ factorial experiment, where $p$ is prime or non prime.

\section{Method}

Consider a matrix $M$ of order $p^{n-1} \times n p$, which represents the construction method of a $p^{n}$ f.e. confounded with a factorial effects as $M=\left[M_{0} M_{1} \ldots \ldots M_{p-1}\right]_{p^{n-1} \times n p}$

where, $M_{u} ; u=0,1, \ldots \ldots \ldots,(p-1)$ resepresent incomplete block defined as,

$M_{u}=\left[V_{1}\left\{p^{0}\right\}, V_{2}\left\{p^{1}\right\}, \ldots \ldots \ldots, V_{n-1}\left\{p^{n-2}\right\}, a_{u}\right]_{p^{n-1} \times n}$

with

$V_{i}\left\{p^{i-1}\right\}=p^{i-1}\left[0 I_{p^{(n-1)-i}}, 1 I_{p^{(n-1)-i}}, \ldots \ldots \ldots,(p-\right.$

1) Ipn-1-i',

each is a column vector of dimension $p^{n-1}$.

$\left\{p^{i-1}\right\}=p^{i-1}$ times repetitions of the elements of $V_{i}$ 's in ascending ordered levels.

$i=1,2,3, \ldots,(n-1)$;

$I_{m}$ : sum vector of dimension $m$; and

$a_{u}=\left[a_{u 1}, a_{u 2}, \ldots, a_{u p^{n-1}}\right]^{\prime}$ is called the adjustment vector.

At $u=0$, the adjustment vector $a_{0}$ is called the key vector and resulting incomplete block represented by this key vector is called the key incomplete block. The key vector $a_{0}$ determines other adjustment vectors $a_{u}$ and hence the incomplete blocks $M_{u}$, for all $u>0$. The elements of the key vector can be obtained by solving the symbolic equation such that $\sum_{i} a_{i} F_{i}+a_{k} F_{k}=0 \bmod p$ where $F_{k}$ represents the adjustment factor.

* Author for correspondence.e-mail: majalil@du.ac.bd 


\section{Limitation of The Method}

This method is applicable in a $p^{n}$ factorial experiment only if $p$ is prime. When $p$ is not a prime number, we cannot apply this method. As for example, suppose the factorial effect $F_{1} F_{2} F_{3} F_{4}$ is confounded in a $3^{4}$ factorial experiment. In this case we can apply this method, because here $p=3$ which is a prime number, but if we want to use this method to confound $F_{1} F_{2} F_{3}^{2}$ in $4^{3}$ factorial experiment considering $F_{3}$ as adjustment factor, we cannot solve the following equation

$$
\sum_{i} a_{i} F_{i}+a_{k} F_{k}=0 \bmod p,
$$

where, $F_{k}$ represents the adjustment factor.

\section{Illustration I}

Suppose the factorial effect $F_{1} F_{2} F_{3} F_{4}$ is to be confounded in a $3^{4}$ factorial experiment. Here, $n=4 ; F_{1}, F_{2}$, $F_{3}$ and $F_{4}$;

$p=3 ; 0,1$ and 2 .

The suggested matrix is

$$
M=\left[M_{0} M_{1} M_{2}\right]_{3^{4-1} \times 4 * 3}=\left[M_{0} M_{1} M_{2}\right]_{27 \times 12}
$$

where,

$M_{u}=\left[V_{1}\left\{p^{0}\right\}, V_{2}\left\{p^{1}\right\}, \ldots \ldots \ldots, V_{n-1}\left\{p^{n-2}\right\}, a_{u}\right]_{p^{n-1} \times n}$

with

$V_{i}\left\{p^{i-1}\right\}=p^{i-1}\left[0 I_{p^{(n-1)-i}}, 1 I_{p^{(n-1)-i}}, \ldots \ldots \ldots,(p-\right.$ 1) $\left.I_{p^{(n-1)-i}}\right]^{\prime}$

each is a column vector of dimension $p^{n-1}$. Therefore, we have,

$M_{0}=\left[V_{1}\left\{3^{0}\right\}, V_{2}\left\{3^{1}\right\}, V_{2}\left\{3^{2}\right\}, a_{0}\right]_{3^{3} \times 4} ;$

$M_{1}=\left[V_{1}\left\{3^{0}\right\}, V_{2}\left\{3^{1}\right\}, V_{2}\left\{3^{2}\right\}, a_{1}\right]_{3^{3} \times 4}$;

$M_{2}=\left[V_{1}\left\{3^{0}\right\}, V_{2}\left\{3^{1}\right\}, V_{2}\left\{3^{2}\right\}, a_{2}\right]_{3^{3} \times 4}$

with

$v_{1}\{1\}=1\left[0 I_{9}, 1 I_{9}, 2 I_{9}\right]_{27 \times 1}^{\prime}$,

$v_{2}\{3\}=3\left[0 I_{3}, 1 I_{3}, 2 I_{3}\right]_{27 \times 1}^{\prime}$,

$v_{3}\{9\}=9\left[0 I_{1}, 1 I_{1}, 2 I_{1}\right]_{27 \times 1}^{\prime}$.

Now the elements of the adjustment vector are obtained from the equation $\sum_{i} a_{i} F_{i}+a_{k} F_{k}=0 \bmod p$, considering $F_{k}=F_{3}$ we have,

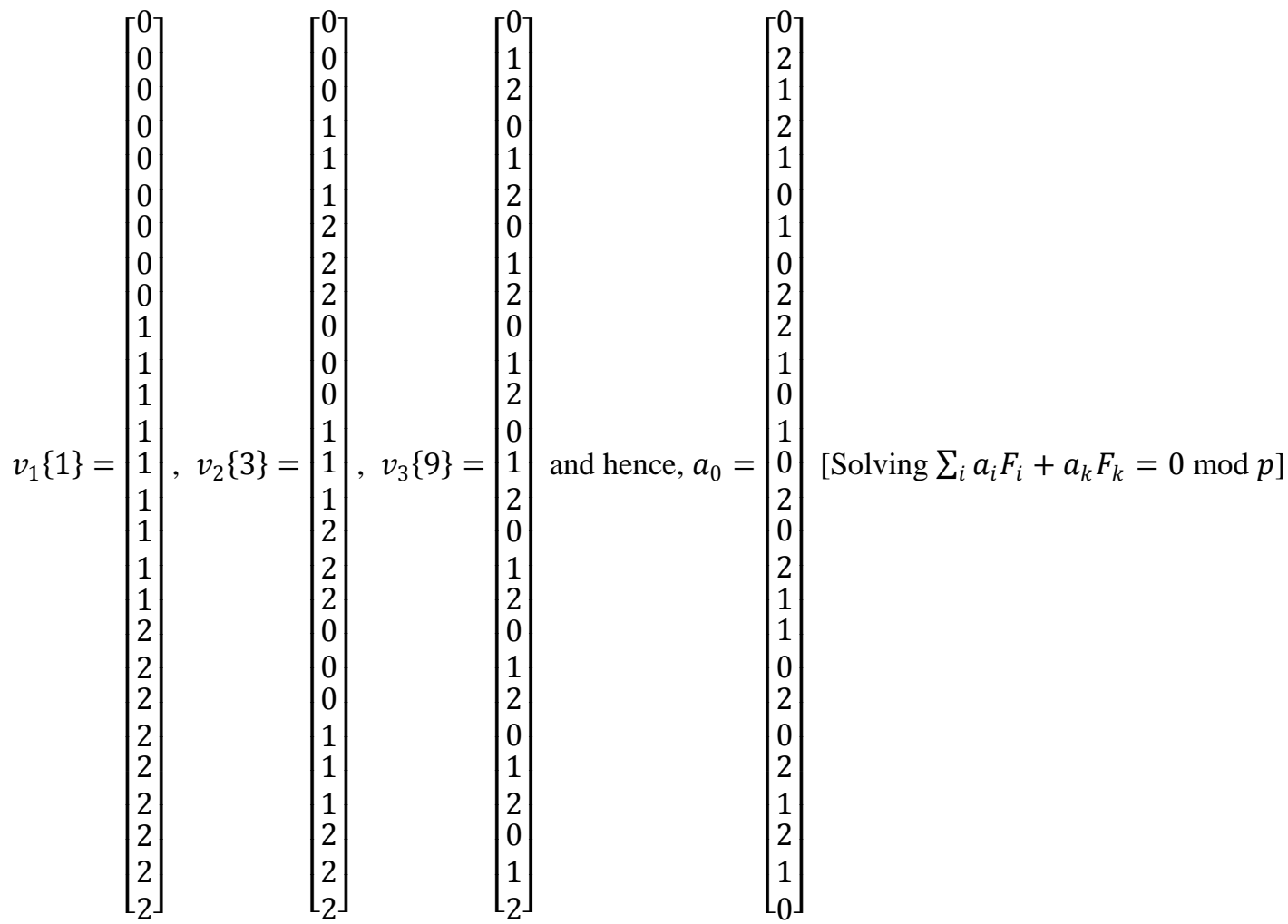

Therefore, 


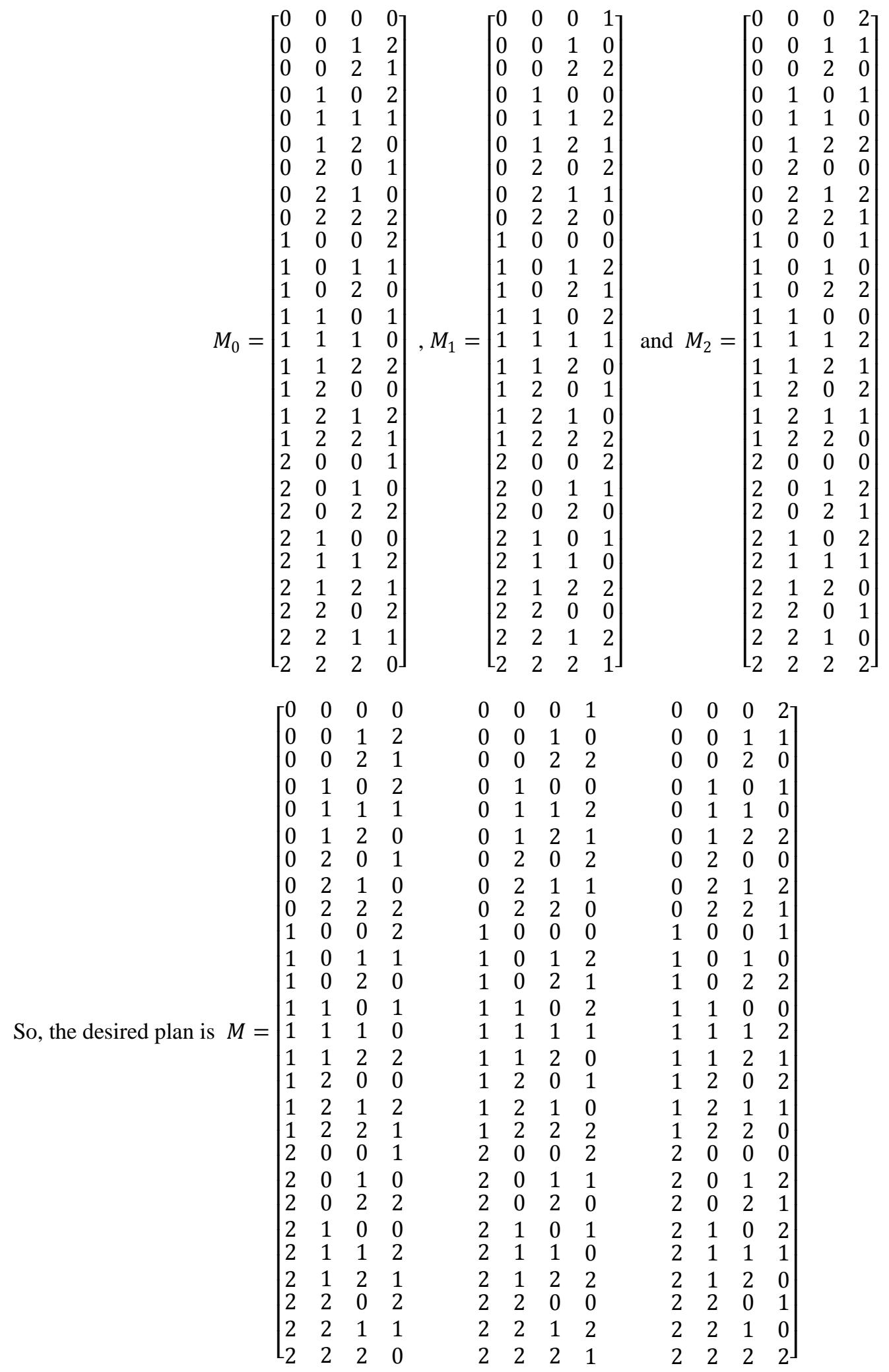

Let us consider the factorial effect $F_{1} F_{2} F_{3}^{2}$ is to be confounded in a $4^{3}$ factorial experiment.

Here, $n=3 ; F_{1}, F_{2}$ and $F_{3}$;

$p=4 ; 0,1,2$ and 3

From the equation, $M=\left[M_{0} M_{1} \ldots \ldots M_{p-1}\right]_{p^{n-1} \times n p}$, we have in this case,

$M=\left[\begin{array}{llll}M_{0} & M_{1} & M_{2} & M_{3}\end{array}\right]_{4^{3-1} \times 3 * 4}$
$=\left[\begin{array}{llll}M_{0} & M_{1} & M_{2} & M_{3}\end{array}\right]_{16 \times 12} ;$

where,

$M_{u}=\left[V_{1}\left\{p^{0}\right\}, V_{2}\left\{p^{1}\right\}, \ldots \ldots \ldots, V_{n-1}\left\{p^{n-2}\right\}, a_{u}\right]_{p^{n-1} \times n}$ with 
$V_{i}\left\{p^{i-1}\right\}=p^{i-1}\left[0 I_{p^{(n-1)-i}}, 1 I_{p^{(n-1)-i}}, \ldots \ldots \ldots,(p-\right.$ 1)Ipn-1-i',

each is a column vector of dimension $p^{n-1}$. Therefore, we have,

$$
\begin{aligned}
& M_{0}=\left[V_{1}\left\{4^{0}\right\}, V_{2}\left\{4^{1}\right\}, a_{0}\right]_{4^{2} \times 3} ; \\
& M_{1}=\left[V_{1}\left\{4^{0}\right\}, V_{2}\left\{4^{1}\right\}, a_{1}\right] 4^{2} \times 3 \text {; } \\
& M_{2}=\left[V_{1}\left\{4^{0}\right\}, V_{2}\left\{4^{1}\right\}, a_{2}\right]_{4^{2} \times 3} \text {; } \\
& M_{3}=\left[V_{1}\left\{4^{0}\right\}, V_{2}\left\{4^{1}\right\}, a_{3}\right]_{4^{2} \times 3} \text {; } \\
& \text { with } \\
& V_{1}\left\{4^{0}\right\}=1\left[0 I_{4}, 1 I_{4}, 2 I_{4}, 3 I_{4}\right]_{16 \times 1}^{\prime} \text {, } \\
& V_{2}\left\{4^{1}\right\}=4\left[0 I_{1}, 1 I_{1}, 2 I_{1}, 3 I_{1}\right]_{16 \times 1}^{\prime} \text {. }
\end{aligned}
$$

Thus,

$$
V_{1}\left\{4^{0}\right\}=\left[\begin{array}{l}
0 \\
0 \\
0 \\
0 \\
1 \\
1 \\
1 \\
1 \\
2 \\
2 \\
2 \\
2 \\
3 \\
3 \\
3 \\
3
\end{array}\right], \quad V_{2}\left\{4^{1}\right\}=\left[\begin{array}{l}
0 \\
1 \\
2 \\
3 \\
0 \\
1 \\
2 \\
3 \\
0 \\
1 \\
2 \\
3 \\
0 \\
1 \\
2 \\
3
\end{array}\right]
$$

Now $a_{u}$ is column vector of dimension 16, i.e.

$$
a_{u}=\left[a_{u 1}, a_{u 2}, \ldots, a_{u 16}\right]
$$

So, to get the elements of the key adjustment vector, $a_{0}$, we have to solve the following equation

$$
x_{1}+x_{2}+2 x_{3}=0(\bmod 4)
$$

Here we consider $F_{3}$ as adjustment factor. Thus we get,

$$
\begin{aligned}
& 0+0+2 x_{3}=0(\bmod 4)==>a_{01}=0 \\
& 0+1+2 x_{3}=0(\bmod 4)
\end{aligned}
$$

We cannot calculate $a_{02}$. Thus we cannot calculate the key adjustment vector, $a_{0}$.

The reason is discussed in the next section.

\section{Reason}

We know that if we multiply an even number by any natural number the result will be an even number. For this reason if we take adjustment factor, $F_{k}$, whose exponent is an even number, we cannot calculate the key adjustment vector $a_{0}$. So we have to take adjustment factor $F_{k}$ whose exponent is odd number.

\section{Moderation}

In this method, proposed by Jalil et. al. $^{12}$, we have to take that factor as adjustment factor whose exponent is odd. If there is more than one factor whose exponents are odd, then any one can be considered as adjustment factor. After moderation, this method can be applied in $p^{n}$ factorial experiments, where $p$ is prime or non prime.

\section{Illustration II}

Construct a confounding plan where the confounded effect is $F_{1} F_{2} F_{3}^{2}$ in a $4^{3}$ factorial experiment.

Solution: Here, $n=3 ; F_{1}, F_{2}$ and $F_{3}$;

$p=4 ; 0,1,2$ and 3

From the equation, $M=\left[M_{0} M_{1} \ldots \ldots M_{p-1}\right]_{p^{n-1} \times n p}$, we

have in this case,

$M=\left[\begin{array}{llll}M_{0} & M_{1} & M_{2} & M_{3}\end{array}\right]_{4^{3-1} \times 3 * 4}$

$=\left[\begin{array}{llll}M_{0} & M_{1} & M_{2} & M_{3}\end{array}\right]_{16 \times 12}$;

where,

$M_{u}=\left[V_{1}\left\{p^{0}\right\}, V_{2}\left\{p^{1}\right\}, \ldots \ldots \ldots, V_{n-1}\left\{p^{n-2}\right\}, a_{u}\right]_{p^{n-1} \times n}$

with

$V_{i}\left\{p^{i-1}\right\}=p^{i-1}\left[0 I_{p^{(n-1)-i}}, 1 I_{p^{(n-1)-i}}, \ldots \ldots \ldots,(p-\right.$

1)Ipn-1-i',

each is a column vector of dimension $p^{n-1}$. Therefore, we have,

$$
\begin{aligned}
& M_{0}=\left[V_{1}\left\{4^{0}\right\}, V_{2}\left\{4^{1}\right\}, a_{0}\right]_{4^{2} \times 3} ; \\
& M_{1}=\left[V_{1}\left\{4^{0}\right\}, V_{2}\left\{4^{1}\right\}, a_{1}\right]_{4^{2} \times 3} ; \\
& M_{2}=\left[V_{1}\left\{4^{0}\right\}, V_{2}\left\{4^{1}\right\}, a_{2}\right]_{4^{2} \times 3} ; \\
& M_{3}=\left[V_{1}\left\{4^{0}\right\}, V_{2}\left\{4^{1}\right\}, a_{3}\right]_{4^{2} \times 3} \\
& \text { with } \\
& V_{1}\left\{4^{0}\right\}=1\left[0 I_{4}, 1 I_{4}, 2 I_{4}, 3 I_{4}\right]_{16 \times 1}^{\prime}, \\
& V_{2}\left\{4^{1}\right\}=4\left[0 I_{1}, 1 I_{1}, 2 I_{1}, 3 I_{1}\right]_{16 \times 1} .
\end{aligned}
$$

Now the elements of the adjustment vector are obtained from the equation $\sum_{i} a_{i} F_{i}+a_{k} F_{k}=0 \bmod p$ where $F_{k}$ represents the adjustment factor. Since the exponents of both $F_{1}$ and $F_{2}$ are odd, anyone can be considered as adjustment factor. Here $F_{1}$ is considered as adjustment factor. Now, we have

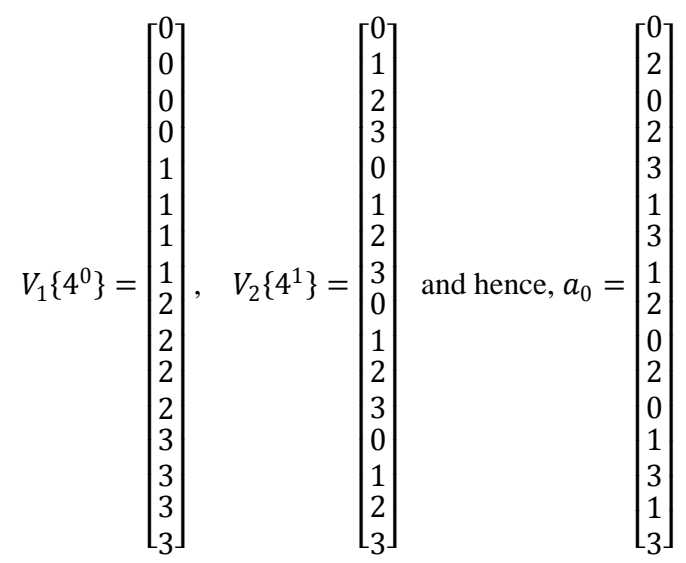

[Solving $\sum_{i} a_{i} F_{i}+a_{k} F_{k}=0 \bmod p$ ].

$a_{1}, a_{2}$ and $a_{3}$ can be obtained by solving $a_{u k}=a_{0 k}+u$ where $k=1,2, \ldots, p^{n-1}$; 
Therefore,

So, the desired plan is

$$
M_{0}=\left[\begin{array}{lll}
0 & 0 & 0 \\
2 & 0 & 1 \\
0 & 0 & 2 \\
2 & 0 & 3 \\
3 & 1 & 0 \\
1 & 1 & 1 \\
3 & 1 & 2 \\
1 & 1 & 3 \\
2 & 2 & 0 \\
0 & 2 & 1 \\
2 & 2 & 2 \\
0 & 2 & 3 \\
1 & 3 & 0 \\
3 & 3 & 1 \\
1 & 3 & 2 \\
3 & 3 & 3
\end{array}\right], \quad M_{1}=\left[\begin{array}{lll}
1 & 0 & 0 \\
3 & 0 & 1 \\
1 & 0 & 2 \\
3 & 0 & 3 \\
0 & 1 & 0 \\
2 & 1 & 1 \\
0 & 1 & 2 \\
2 & 1 & 3 \\
3 & 2 & 0 \\
1 & 2 & 1 \\
3 & 2 & 2 \\
1 & 2 & 3 \\
2 & 3 & 0 \\
0 & 3 & 1 \\
2 & 3 & 2 \\
0 & 3 & 3
\end{array}\right]
$$

$$
M_{2}=\left[\begin{array}{lll}
2 & 0 & 0 \\
0 & 0 & 1 \\
2 & 0 & 2 \\
0 & 0 & 3 \\
1 & 1 & 0 \\
3 & 1 & 1 \\
1 & 1 & 2 \\
3 & 1 & 3 \\
0 & 2 & 0 \\
2 & 2 & 1 \\
0 & 2 & 2 \\
2 & 2 & 3 \\
3 & 3 & 0 \\
1 & 3 & 1 \\
3 & 3 & 2 \\
1 & 3 & 3
\end{array}\right], \quad M_{3}=\left[\begin{array}{lll}
3 & 0 & 0 \\
1 & 0 & 1 \\
3 & 0 & 2 \\
1 & 0 & 3 \\
2 & 1 & 0 \\
0 & 1 & 1 \\
2 & 1 & 2 \\
0 & 1 & 3 \\
1 & 2 & 0 \\
3 & 2 & 1 \\
1 & 2 & 2 \\
3 & 2 & 3 \\
0 & 3 & 0 \\
2 & 3 & 1 \\
0 & 3 & 2 \\
2 & 3 & 3
\end{array}\right]
$$

$\left.\begin{array}{llllll}2 & 0 & 0 & 3 & 0 & 0 \\ 0 & 0 & 1 & 1 & 0 & 1 \\ 2 & 0 & 2 & 3 & 0 & 2 \\ 0 & 0 & 3 & 1 & 0 & 3 \\ 1 & 1 & 0 & 2 & 1 & 0 \\ 3 & 1 & 1 & 0 & 1 & 1 \\ 1 & 1 & 2 & 2 & 1 & 2 \\ 3 & 1 & 3 & 0 & 1 & 3 \\ 0 & 2 & 0 & 1 & 2 & 0 \\ 2 & 2 & 1 & 3 & 2 & 1 \\ 0 & 2 & 2 & 1 & 2 & 2 \\ 2 & 2 & 3 & 3 & 2 & 3 \\ 3 & 3 & 0 & 0 & 3 & 0 \\ 1 & 3 & 1 & 2 & 3 & 1 \\ 3 & 3 & 2 & 0 & 3 & 2 \\ 1 & 3 & 3 & 2 & 3 & 3\end{array}\right]$

5. Kempthorne, O., 1952. The design and Analysis of Experiments. John Wiley \& Sons, Inc., New York.

6. Das, M. N., 1964. A Somewhat Alternative Approach for Construction of Symmetrical Factorial Designs and Obtaining Maximum Numbers of Factors. Calcutta Statistical Association Bulletin, 13, 1-17.

7. Cotter, S. C., 1974. A General Method of Confounding for Symmetrical Factorial Experiments. JRSS, 36, 267-276.

8. Dean, A. M. and J. A. John, 1975. Single Replicate Factorial Experiments in Generalized Cyclic Design: Symmetrical Arrangements. JRSS, B 37, 63-71.

9. Patterson, H. D., 1976. Generation of Factorial Designs. JRSS, B 37, 175-179.

10. Mallick, S. A., 1973. A System of Simultaneous Confounding of Two Effects in A $3^{n}$ - Factorial Design. Dacca University Studies, Pt. B. XXI, 33-36.

11. Mallick, S. A., 1975. A System of Simultaneous Confounding of Two Effects in A $4^{n}$ - Factorial Design. Dacca University Studies, Pt. B. XXIII (1), 7-10.

12. Jalil, M. A., S. A Mallick and M. S. Rahman, 1990. Matrix Method of Designing Layout in $p^{n}$ - Factorial Experiment. Dhaka University Studies, B, 38(2), 123-128.
4. Kempthorne, O., 1947. A Simple Approach to Confounding and Fractional Replication in Factorial Experiments. Biometrika, 34, 255-272. 